\title{
Venous Thromboembolism in COVID-19: Are Women Different?
}

\author{
Shibba Takkar Chhabra Prerna Goyal ${ }^{2, \bullet}$ \\ ${ }^{1}$ Department of Cardiology, Dayanand Medical College and \\ Hospital, Unit Hero DMC Heart Institute, Ludhiana, Punjab, India \\ ${ }^{2}$ Department of Internal Medicine, RG Stone and Superspeciality \\ Hospital, Ludhiana, Punjab, India \\ Ind J Car Dis Wom:2020;5:200-208
}

\begin{abstract}
Address for correspondence Shibba Takkar Chhabra, MD, DM, Department of Cardiology, Dayanand Medical College and Hospital, Unit Hero DMC Heart Institute, Ludhiana, Punjab, India (e-mail: shibbachhabra@yahoo.com).
\end{abstract}

\begin{abstract}
Keywords

- COVID

- thromboembolism

- thromboprophylaxis

- venous

- women

The epidemiological data suggests predominant male prevalence, morbidity and mortality with SARS COVID-2. Similarly, venous thromboembolic (VTE) events have a male sex predilection with variant mechanisms involving angiotensin-converting enzyme 2 (ACE-2) expression and pathways in women. COVID-19 could directly affect or it could be an indirect action of the disease via critical ailment hypoxemia, or hemostatic abnormalities might be the underlying mechanisms of VTE in a COVID-19 patient with baseline risk factor profile. VTE diagnosis in a COVID scenario has issues of a prone positioned patient, exposure of health workers and minimal therapeutic benefits in a critically ill patient with acute respiratory distress syndrome (ARDS). Anticoagulation with low-molecular weight heparin (LMWH) can be chosen over unfractionated heparin (UFH) with less monitoring requirements and thereby low exposure to healthcare workers. Variant guidelines for thromboprophylaxis (in hospital/extended postdischarge) have come up, stating anticoagulant administration, according to baseline risk profile and hemostatic biomarkers. Catheter-directed interventions should be reserved only for life-threatening situations. In women, hormonal milieu (for e.g., $17 \beta$-estradiol) might influence occurrence of favorable ACE 2 polymorphisms with less VTE events. The management strategies in a female patient with VTE would be more or less similar to males. Combined oral contraceptives (COC) and estrogen replacement therapies (ERT) may be curtailed in COVID-19 positive patients, given their thrombogenic potential. Pregnancy and postpartum state in COVID-19 positive patient need VTE prophylaxis all the more in the presence of risk profile favoring VTE. Also, VTE prophylaxis when indicated should be continued in women in a normal menstrual cycle. Bleeding risks specific to women (menorrhagia secondary to dysfunctional uterine bleeding [DUB], fibroids, adnexal malignancies and masses, hypothyroidism, etc.) need to be weighed while taking a decision for indicated anticoagulation regimen.
\end{abstract}

\section{Introduction}

The epidemiological data suggests predominant male prevalence, morbidity and mortality with SARS COVID-2. ${ }^{1}$ Similarly, venous thromboembolic (VTE) events have a male sex predilection with variant mechanisms involving angiotensin-converting enzyme 2 (ACE-2) expression and pathways. ${ }^{2}$ The present review aims to analyze the burden, mechanism and management strategies studied so far in the COVID-19 pandemic. It also attempts to visualize the mechanisms and variant management strategies of VTE events in women versus men.

The COVID-19 pandemic can be responsible for VTE disease via several mechanisms. These mechanisms could be a candid action of COVID-19, an implicit effect of the disease
(C)2020 Women in Cardiology and Related Sciences
License terms

(1) (1) $\odot \circledast$ 
via critical ailment hypoxemia, or hemostatic abnormalities which might ultimately predispose the patient to disseminated intravascular coagulation (DIC). Moreover, SARS, critical illness and baseline risk factor profile might predispose a patient to developing VTE.

The novel antitherapeutic therapy of COVID-19 might interact with antiplatelets and anticoagulants in the patients with thromboembolic disease. In a retrospective analysis by Tang et al $^{3}$ among 183 patients with COVID-19, an overall mortality of $11.5 \%$ was observed. This era might also affect the care and management of the patients who do not have COVID-19 but have pre-existing thrombotic or thromboembolic ailments.

VTE events have a lesser predilection for fair sex. Variant biology, hormonal milieu, menstrual phases, and select bleeding risks are issues specific to women. Although the management strategies and guidelines do not differentiate between the two sexes with regard to VTE, pertinent issues need an addressal in women. After presenting the baseline data for VTE in COVID-19, this review discusses the issues different and special to women prior to conclusion.

\section{Hemostasis Markers}

Mild thrombocytopenia and increased D-dimer values are the usual hematological parameters noticed in COVID-19 positive subjects. Besides D-dimer, prolonged prothrombin time, international normalized ratio (INR) and thrombin time have also represented the intensity of disease severity. ${ }^{4}$ Cytokine storm in a patient with COVID-19 predisposes to a SARS-like condition.

Raised D dimer has been linked with poor prognosis and an increased incidence of needing mechanical ventilation and ICU care and mortality in patients with COVID-19. ${ }^{5}$ As much as $71.4 \%$ deaths in a study had criteria for DIC. In these patients, 3.5- and 1.94-fold rise in D-dimer and fibrin degradation products (FDP) was reported. The prothrombin time (PT) was also prolonged in the nonsurvivors by $14 \%$ ( $p$ value $<0.001$ ). ${ }^{3}$ In yet another study from Netherlands, $31 \%$ of the 184 ICU admitted critical patients had thrombotic events, most commonly VTE. ${ }^{4}$

Whether the hematological abnormalities noted in SARS-COV-2 patient are a cause or result of the cytokine storm is still being evaluated. Moreover, hepatic dysfunction in these patients might lead to changes in hemostatic parameters. The role of antiphospholipid antibodies predisposing to thrombotic events in a patient with COVID-19 is also being studied.

\section{Pathophysiology of VTE in COVID}

The pathophysiology of VTE in a patient with SARS-COV-2 infection can be studied under the headings: base line risk profile of the patient, hematological abnormalities, and clinical manifestations.

\section{Baseline Risk Profile}

The baseline risk profile of the patient might be inclusive of several risk factors predisposing to VTE diseases. These risk factors could include a patient admitted with acute critical illness, a bedridden state predisposing to stasis, underlying genetic abnormalities, fever, gastroenteritis, sepsis, hepatic injury, underlying chronic kidney disease or chronic obstructive airway diseases, underlying heart failure, or malignancies predisposing to VTE. In female gender, the state of pregnancy and postpartum states are particularly noted to be hypercoagulable, especially in presence of other VTE risk factors. Moreover, women on combined oral contraceptives (COC) and estrogen replacement therapy (ERT) have aggravated risk of VTE. COC use is associated with 2 to 6 folds increase in VTE risk, while in pregnancy, the risk increases by 4 to 5 folds.

\section{Hematological Abnormalities}

In a subject infected with SARS-COV-2, underlying risk factors might lead to activation of inflammatory mediators. Inflammatory cytokines like interleukin-6 and C-reactive protein (CRP) might further increase with a predisposition to developing superimposed infections. Endothelial dysfunction and haemostatic activation might follow this activated inflammatory response. Increase in tissue factor, Von Willebrand factor and hemostatic abnormalities like lymphopenia and thrombocytopenia might ensue. Hepatic involvement might lead to decreased coagulation and antithrombin formation, thereby predisposing to thrombotic abnormalities.

\section{Clinical Manifestations}

These hemostatic abnormalities ultimately culminate into VTE events like pulmonary micro or macro thromboembolism and intravascular coagulopathy. Moreover, an elevation in cardiac biomarkers like troponins and myocardial injury also ensue. VTE risk has been reported to be maximal in patients who are critically ill and hospitalized. The risk estimates in COVID-19 outpatients are not yet available.

\section{VTE Prophylaxis in COVID-19}

Wang et $\mathrm{al}^{6}$ highlighted the importance of VTE prophylaxis in COVID-19 patients. They reported increased VTE risk in up to $40 \%$ of their hospitalized COVID-19 patients. ${ }^{6}$ Administration of all scheduled doses of VTE prophylaxis should be ensured to avoid untoward events with worse clinical outcomes. A daily dosing regime of low-molecular weight heparin (LMWH) has an advantage over unfractionated heparin (UFH) with twice daily doses in COVID 19 by reducing the use of PPE and exposing the COVID-19 healthcare workers (HCWs). Daily LMWH or subcutaneous UFH has been advocated by WHO in admitted COVID-19 subjects. Mechanical VTE prophylaxis via intermittent pneumatic compression could be considered in subjects (especially in sedentary ones) with contraindications to prophylactic anticoagulation., ${ }^{4,7}$

In pregnant and postpartum patients with COVID-19, although the data is limited, it can be assumed that they are predisposed to VTE. ${ }^{8,9}$ Risk assessment of a pregnant patient with COVID-19 is prudent, and pharmacological thromboprophylaxis in these pregnant patients with SARS-COV-2 should be considered all the more in the presence of baseline increased VTE risk. 
The data specific to COVID-19 with regard to extended VTE prophylaxis is minimal. Hence, in patients with SARS-COV-2 infection, individualization of the treatment and weighing the risk and benefits of the tendency to developing VTE versus the hemorrhagic risk of patient needs to be done. A prolonged and extended prophylaxis for up till 45 days might be offered to COVID-19 subjects with elevated VTE risk, for example, patients with comorbidities like active cancer, patients with decreased mobility and according to expert opinion in some cases more than twice the increase in upper limit of D-dimer values with low-bleeding risk and increased body mass index (BMI) $\left(\geq 30 \mathrm{~kg} / \mathrm{m}^{2}\right)$.

In mild COVID-19 subjects in quarantine, the role of pharmacological prophylaxis is yet uncertain; hence mobility at home should be recommended. The pharmacological prophylaxis in these patients could be reserved for high-risk VTE subjects, for instance, in those patients who have reduced mobility, a previous history of thromboembolic event or in those who have active ongoing malignancy. ${ }^{4}$

\section{Diagnosis of Venous Thromboembolism (VTE)}

Raised D-dimer, a marker of severity of COVID-19, might not routinely indicate an underlying VTE process. ${ }^{10} \mathrm{~A}$ VTE event should be suspected in a patient of COVID-19 when the subject develops typical deep vein thrombosis (DVT) symptoms, hypoxemia which is out of proportion to the underlying respiratory pathology or development of acute unexplained right ventricular (RV) dysfunction. The diagnostic challenges in a patient with COVID-19 for VTE are as follows

1. Infection spreading to HCWs and inpatients via an unstable COVID-19 subject.

2. Prone position in a patient with severe acute respiratory distress syndrome (ARDS) limiting the imaging studies.

3. Critical ailment and prone position not permitting the required investigations for DVT.

4. Grave prognosis of the patient with COVID-19 with ARDS requiring prone position might not benefit with any other therapeutic intervention, even if underlying VTE is diagnosed.

Echocardiography might be considered to look for worsening RV dysfunction and diagnosis of RV clots/clot in transit in COVID-19 subjects. ${ }^{11}$

\section{Empiric Therapeutic Anticoagulation}

In a single-center small sampled Chinese study, D-dimer more than $1500 \mathrm{~g}$ per $\mathrm{mL}$ was believed to have sensitivity of 85 and specificity of $88.5 \%$ for detection of VTE episodes. ${ }^{4}$ Prophylactic anticoagulation is still preferred over therapeutic anticoagulation (intermediate or full dose) in subjects with yet unconfirmed VTE. The optimal anticoagulant dose has not yet been determined, although suggestions of benefit exist across some studies.

Tang et al stated a decrease mortality in patients on anticoagulant treatment with severe COVID-19 manifestations. ${ }^{12}$ Incident VTE of $25 \%$ and $31 \%$ in severe COVID patients in two studies from China and Netherlands has been reported, respectively. While the study from China noted that VTE prophylaxis was not administered to any of these patients, in the other study, all the incident VTE patients were administered prophylactic anticoagulation, although lower dosing was an observation in some subjects. Under diagnosis of VTE in severe COVID-19 subjects is possible, especially when ARDS might itself potentiate hypoxic pulmonary vasoconstriction, pulmonary arterial hypertension followed by RV dysfunction.

\section{Therapeutic Anticoagulation in Patients with Diagnosed Venous Thromboembolism}

Once the diagnosis of VTE is confirmed, the choice of therapeutic anticoagulant would be determined by renal, hepatic, and gastrointestinal functions in a given patient as well as hematological parameters, for example, any associated thrombocytopenia and deranged haemostatic markers. UFH might be preferred by several physicians in hospital settings, given its transient effects and rare drug interactions with upcoming COVID-19 therapy. However, maintenance of therapeutic activated partial thromboplastin time (APTT) ratio with UFH requires recurrent HCW exposure to the patients; hence, LMWH could be the choice. NOACs could be preferred in these patients with their benefits of lack of need of monitoring and ability to continue at equivalent doses at times of discharge. However, drug interactions of novel oral anticoagulants (NOACs) can be an issue, for instance, apixaban and betrixaban might need doses to be adjusted in COVID-19 patients being administered lopinavir and ritonavir. Edoxaban and rivaroxaban should not be administered together with these agents due to potential interactions. Once stabilized, the patients of COVID-19 can be administered NOACs or LMWH at the discharge. This would help in limiting the need for recurrent INR monitoring and exposure of HCWs. Also, in organ dysfunction in a patient with severe COVID-19, the use of these drugs can be tedious, especially due to lack of effective reversal agents.

\section{Catheter-Directed Therapy for VTE}

In a given patient with COVID-19 along with acute DVT, anticoagulation with home treatment, wherever feasible, should be encouraged. Endovascular therapies with local fibrinolysis or embolectomy should be reserved in occasional cases with phlegmasia or truly refractory symptoms.

Similarly, in patients with intermediate risk hemodynamically stable pulmonary embolism (PE), anticoagulation with close monitoring should be encouraged. Rescue systemic fibrinolysis may be considered in patients with further deterioration or hemodynamically unstable high-risk patients with massive PE. Catheter-based therapies should be reserved only in the cases where systemic fibrinolysis may not be feasible in the settings where infection control is well-equipped, appropriate and equivalent. Bedside extracorporeal membrane oxygenation (ECMO) might be considered in COVID-19 positive patients rather than utilization of 
interventional modalities needing cardiac catheterization laboratories or operating rooms. ${ }^{13}$

The use of inferior vena cava (IVC) filters should be minimized to cases with recurrent PE in spite of adequate anticoagulation or in those with absolute contraindication to anticoagulation.

\section{Approach to Non COVID-19 Patients with VTE}

In patients without COVID-19 and acute VTE, outpatient management or an early discharge should be preferred. Telemedicine could be opted methodology of follow-up. With regard to pharmacotherapy, these patients could be maintained on NOACs or LMWH to avoid recurrent contact with HCWs for maintenance of INRs in patient receiving vitamin $\mathrm{K}$ antagonizes.

In patients already receiving vitamin $\mathrm{K}$ antagonizes, extending INR testing in intervals could be suggested if prior INRs have been stable. Home-based INR checks or drive through INR testing should be encouraged, although switching to a NOACs or LMWH would be the clinically appropriate strategy wherever feasible.

\section{Synopsis of Guidelines and Position Papers on Venous Thromboembolism}

Standard dose thromboprophylaxis in hospitalized COVID-19 patients has been advocated by Chinese (CMDA), ${ }^{14}$ ISTH, ${ }^{15}$ and Thrombosis-UK ${ }^{16}$ recommendations. However, intensified anticoagulation varying with disease severity (clinically/ hematological or other biological parameters) has been recommended by CCS, ${ }^{17} \mathrm{GIHP}, \mathrm{GFHT},{ }^{18} \mathrm{NIPHN},{ }^{19} \mathrm{GTH},{ }^{20} \mathrm{SSC}^{21}$ and Swiss Haematology ${ }^{22}$ Society.

Full-dose therapeutic anticoagulation based on inflammatory markers in oxygen-dependent patients or patients documenting rising D-dimer on prophylactic treatment has been recommended by four guidelines.

ASH guidelines, ${ }^{23}$ the CMDA,${ }^{14}$ the $\mathrm{CCS}^{17}$ recommendations, the GTH ${ }^{24}$ SISET, ${ }^{25}$ and the ISTH/NATF/ESVM/IUA ${ }^{26}$ position paper recommend extended prophylaxis postdischarge up to 45 days in high-risk VTE subjects with a low-bleeding risk. ${ }^{25}$ Standard dose thromboprophylaxis is recommended in outpatient COVID-19 patients with multiple VTE risk factors by the SISET and the GTH guidelines. The ISTH/NATF/ESVM/ IUA consensus paper suggests individualization of thromboprophylaxis in patients with increased VTE risk without high-bleeding risk (-Table $\mathbf{1}$ ).

Although the guidelines do not differentiate between male and female sex with regard to VTE management, issues pertinent to female sex need to be addressed. A hypercoagulable state of pregnancy and postpartum period warranting adequate thromboprophylaxis need special consideration, especially in the presence of other VTE risk factors. VTE prophylaxis when indicated should be continued in women in a normal menstrual cycle. Bleeding risks specific to women (menorrhagia secondary to dysfunctional uterine bleeding [DUB], fibroids, adnexal malignancies and masses, hypothyroidism, etc.) need to be weighed while taking a decision for indicated anticoagulation regimen.

\section{COVID-19 Positive Women with Venous Thromboembolism-What's Different?}

The SARS COVID-2 pandemic has so far appeared to have higher death rate in the presence of comorbidities and among men. Higher prevalence of morbidity and mortality in men raises a possibility of sex-dependent susceptibility. Males could be more susceptible to SARS COVID-19 disease due to variant involvement of ACE-2. ACE-2, when activated by the spike protein of SARS COVID-2 virus, is associated with the penetration of the same into the epithelial cells and myocardium. ${ }^{2} 17 \beta$ estradiol has been known to increase the expression and activity of this enzyme in adipose tissue as well as the kidneys. ${ }^{27}$ Higher myocardial ACE-2 expression has been seen in the male hypertensive mice which resolves soon after orchiectomy. ${ }^{28}$

An increased mortality with VTE events has been known to be associated with COVID-19, especially in the critically sick ICU patients. The mortality, which is secondary to VTE events, is even more in the hypogonadal men on treatment with testosterone, especially in patients with greater genetic predisposition. Even further emerging are the epidemiological aspects of a variant expression of ACE-2 under the influence of sex hormones. ACE-2 is expressed in both mouse and human adult Leydig cells but in a testosterone-independent manner. ACE-2 has its role in ovarian granulosa cells with its expression rising with a rise in LH. A higher mortality has been reported in men due to higher cardiovascular and myocardial involvement secondary to the viral infection with COVID-19 via the ACE-2 pathway. Hypothesis of ACE-2 expression in the myocardium being modulated by androgen and the role of androgen receptor gene polymorphisms cannot be ruled out in the COVID-19 positive male patients..$^{28-30}$

Similarly, the concern of increased VTE events in the patients receiving the testosterone is rising. A recent crossover study with 39622 men, with 3110 having hypogonadism was done. ${ }^{29} \mathrm{~A}$ higher VTE risk was found in males, with and without hypogonadism, who were on testosterone replacement therapy. The risk was all the more higher in patients with Klinefelter syndrome. ${ }^{30}$ While testosterone administration needs to be halted in patients in the era of SARS COVID-2 pandemic, given its association with increased VTE events which could be lethal, there is a suggestion of considering ERT in hypogonadal and postmenopausal women. This could also suggest the involvement of polymorphisms of androgen receptor activator and repressor proteins ${ }^{31}$ and the role of selective androgen receptor modulators (SERM) as therapeutic agents in men in the era of the COVID-19 pandemic. ${ }^{32}$

Barring one study by Cui et al, ${ }^{33}$ in which a female predilection for VTE was found to the tune of $64 \%$ in women versus $46 \%$ men getting affected, almost all the other studies have shown a higher predilection of VTE events in the male population. Spiezia et $\mathrm{al}^{34}$ and Ranucci et $\mathrm{a}^{35}$ reported $91 \%$ and $94 \%$ of subjects having VTE as males, whereas most of the other studies reported 60 to $80 \%$ of their subjects with COVID-19 having VTE as men. ${ }^{36}$

The management strategies in a female patient with VTE would be more or less similar to males. However, pregnancy and postpartum state in women could be a consideration, 


\begin{tabular}{|c|c|c|c|c|c|c|c|c|c|c|c|}
\hline 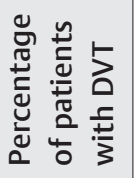 & $\begin{array}{l}\stackrel{\circ}{\circ} \\
\stackrel{d}{d}\end{array}$ & 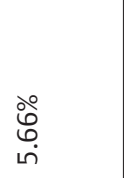 & $\frac{\pi}{L}$ & ১े & 仓ัڤ & 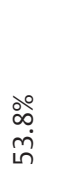 & 总 & $\begin{array}{l}\stackrel{\circ}{6} \\
\stackrel{+}{+}\end{array}$ & 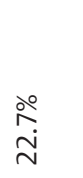 & 0 & $\stackrel{\circ}{\stackrel{\circ}{\zeta}}$ \\
\hline 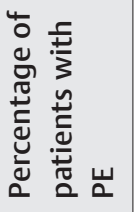 & $\frac{s}{z}$ & $\frac{\Sigma}{z}$ & 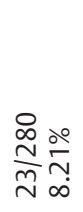 & 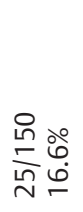 & 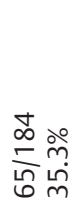 & $\begin{array}{l}\stackrel{20}{\hat{~}} \\
\stackrel{N}{\sigma} \\
\end{array}$ & $\begin{array}{l}\widehat{O} \\
\text { mo } \\
\text { 응 }\end{array}$ & $\begin{array}{l}\hat{O} \text { 울 } \\
\text { 충 }\end{array}$ & 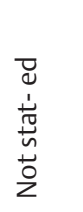 & $\frac{0}{\sigma_{0}}$ & $\begin{array}{l}\text { 융 } \\
\text { ஸn }\end{array}$ \\
\hline 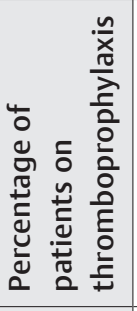 & ஓे & $\frac{\pi}{x}$ & $\frac{\pi}{x}$ & 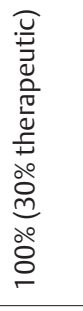 & ○ें & 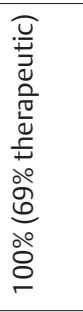 & 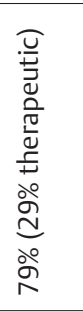 & 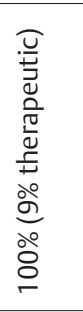 & ○े & ○े & ○े \\
\hline $\begin{array}{l}\text { Ф̃ } \\
\text { Ė } \\
\vdots\end{array}$ & ஓें & ํํํ & $\frac{\pi}{z}$ & ڤํ & $\stackrel{\stackrel{\circ}{\sim}}{\sim}$ & $\stackrel{\circ}{\text { ஸे }}$ & ฌે & $\frac{\circ}{\dot{\gamma}}$ & ळे & ๖̊ & $\frac{\pi}{z}$ \\
\hline$\sum^{\frac{c}{0}}$ & 仓े & ํํㅅ & $\frac{\pi}{z}$ & $\stackrel{\circ}{\infty}$ & 仓̊ํํ & $\stackrel{\circ}{\stackrel{2}{人}}$ & ळे & ڤे & ڤั & ஓें & $\frac{s}{z}$ \\
\hline 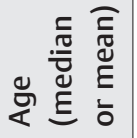 & 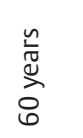 & $\begin{array}{l}\frac{n}{\sqrt[n]{J}} \\
\stackrel{\vec{J}}{\vec{J}}\end{array}$ & $\frac{\pi}{c}$ & 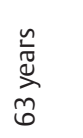 & 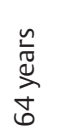 & 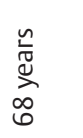 & $\begin{array}{l}\frac{n}{0} \\
\stackrel{0}{0} \\
\stackrel{0}{8}\end{array}$ & 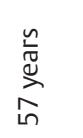 & 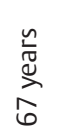 & 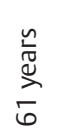 & ถి \\
\hline$z$ & $\bar{\infty}$ & กิ & $\stackrel{\circ}{\sim}$ & $\stackrel{\text { 으 }}{r}$ & $\stackrel{+}{\infty}$ & $\stackrel{\bullet}{\sim}$ & $\begin{array}{l}\infty \\
m \\
m\end{array}$ & $\stackrel{ }{\circ}$ & $\approx$ & $\stackrel{6}{\leftarrow}$ & $\ddot{\sigma}$ \\
\hline 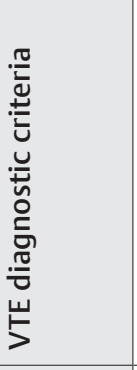 & 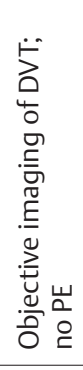 & 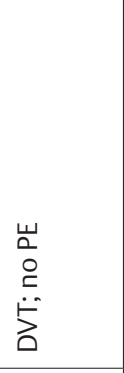 & 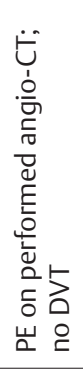 & 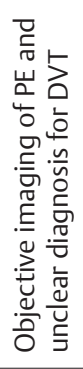 & 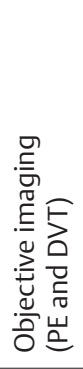 & 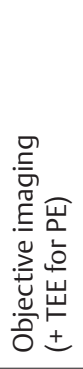 & 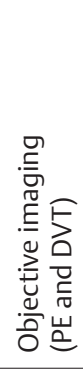 & 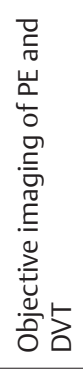 & 5 & 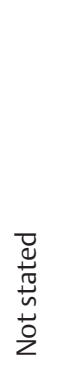 & 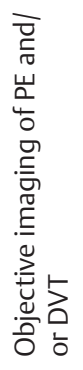 \\
\hline 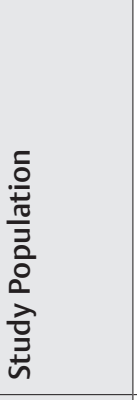 & 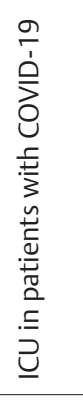 & 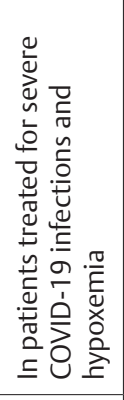 & 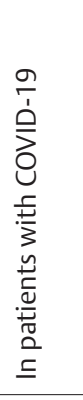 & 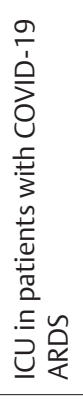 & 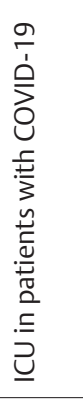 & 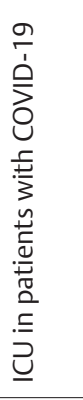 & 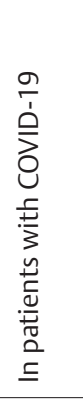 & 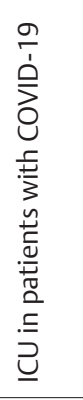 & 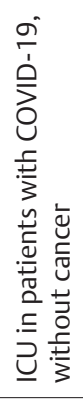 & 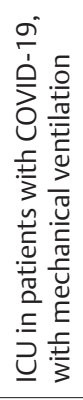 & 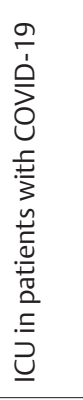 \\
\hline 总 & 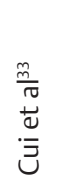 & 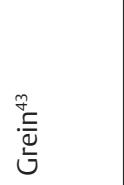 & 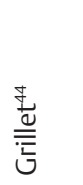 & $\begin{array}{l}\frac{0}{\pi} \\
\frac{0}{0} \\
\tilde{U} \\
\frac{E}{0} \\
\frac{a}{I}\end{array}$ & 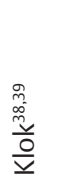 & 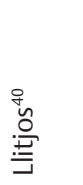 & 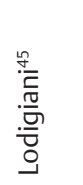 & $\frac{\sqrt{\frac{\alpha}{0}}}{2}$ & 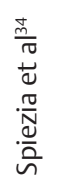 & 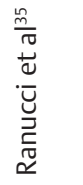 & 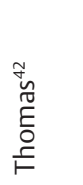 \\
\hline
\end{tabular}




\begin{tabular}{|c|c|c|c|c|c|c|}
\hline 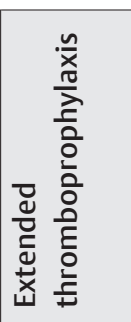 & 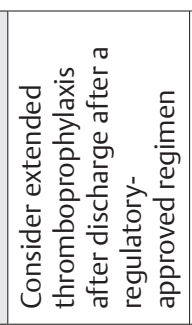 & 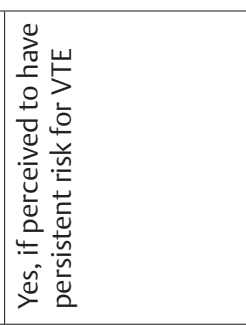 & 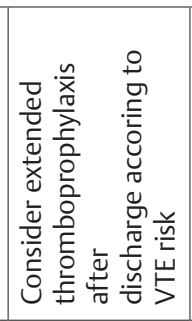 & $\frac{\pi}{C}$ & 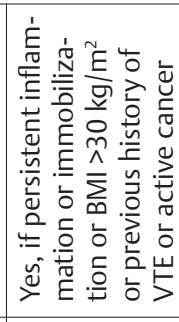 & $\frac{s}{z}$ \\
\hline 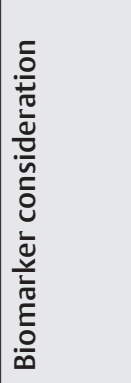 & $\frac{s}{z}$ & 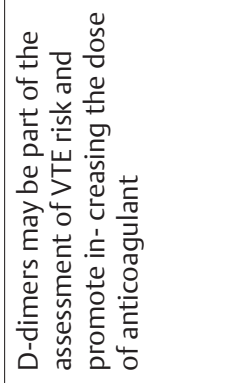 & z & 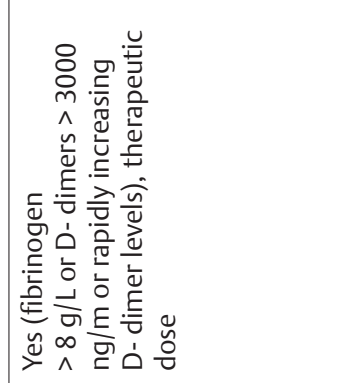 & 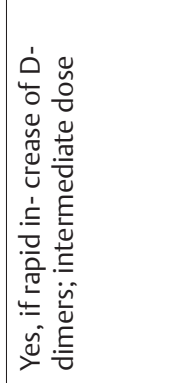 & 2 \\
\hline 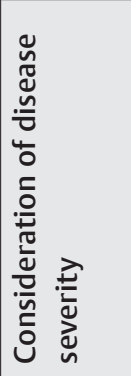 & z & 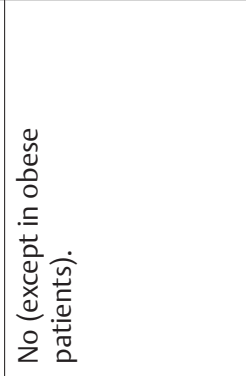 & 2 & 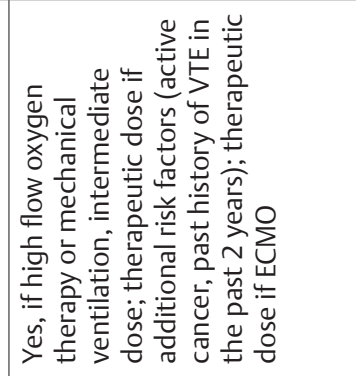 & 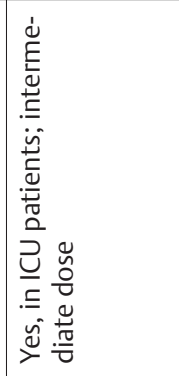 & $\frac{s}{z}$ \\
\hline 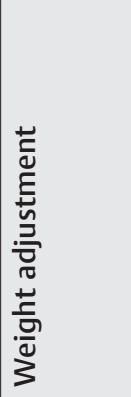 & 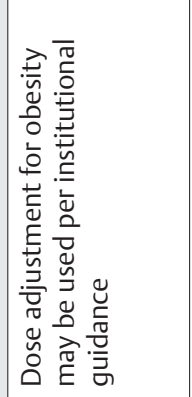 & 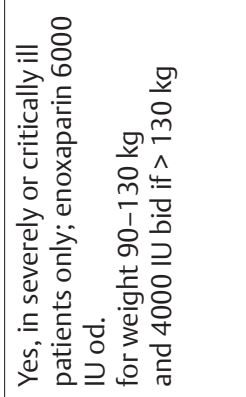 & $\frac{\Sigma}{z}$ & 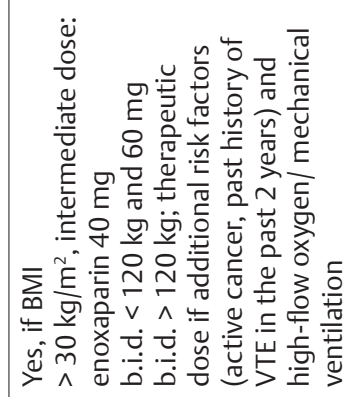 & 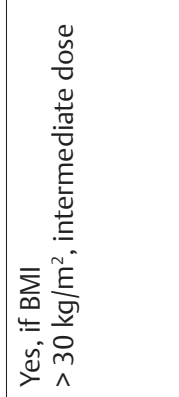 & $\frac{s}{z}$ \\
\hline 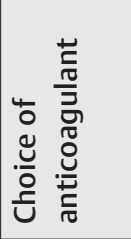 & 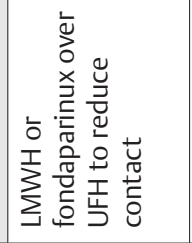 & 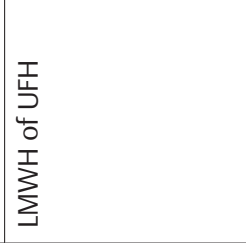 & 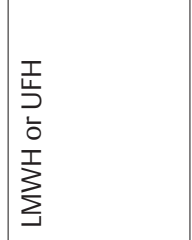 & 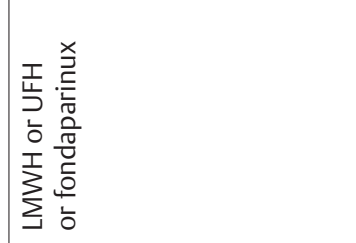 & $\sum_{\sum}^{I}$ & $\sum_{\sum}^{T}$ \\
\hline 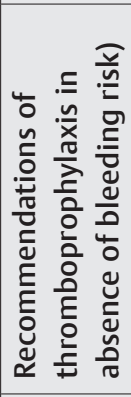 & 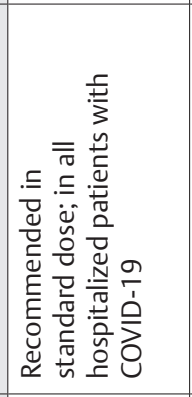 & 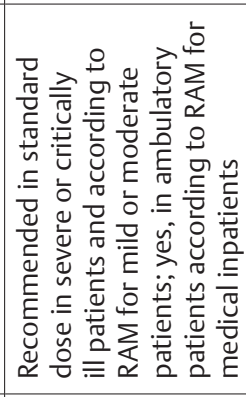 & 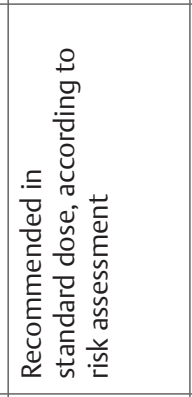 & 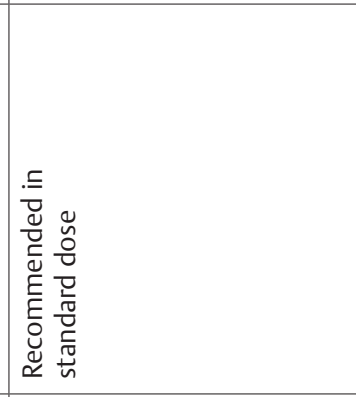 & 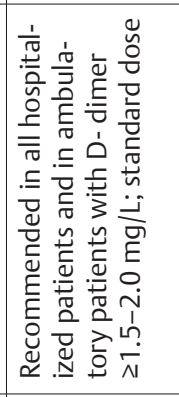 & 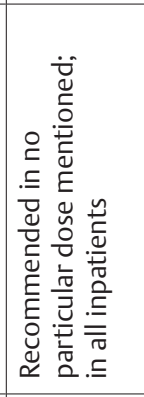 \\
\hline 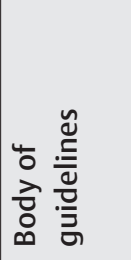 & 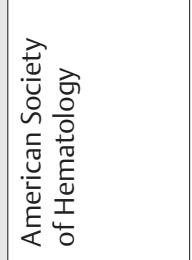 & 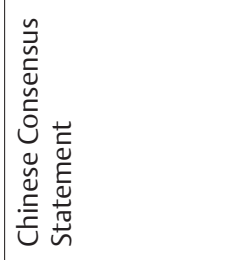 & 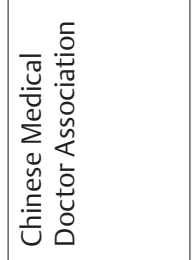 & 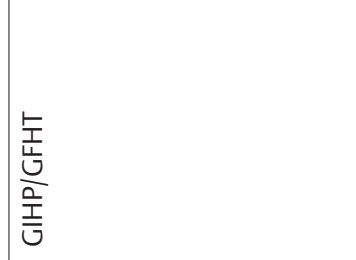 & 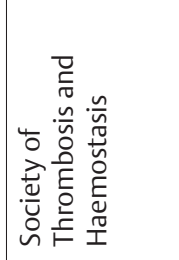 & 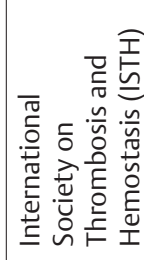 \\
\hline
\end{tabular}




\begin{tabular}{|c|c|c|c|c|c|c|}
\hline 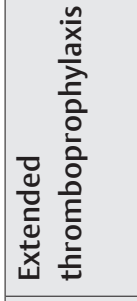 & 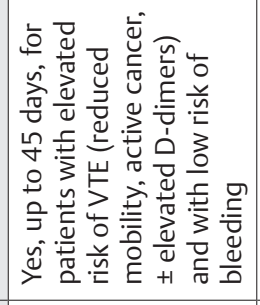 & $\frac{\pi}{z}$ & 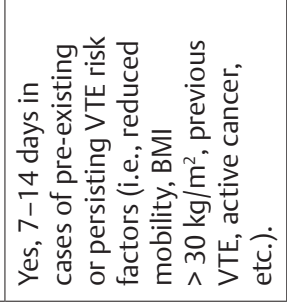 & 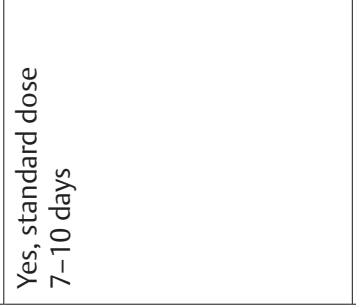 & $\frac{s}{z}$ & $\frac{s}{z}$ \\
\hline 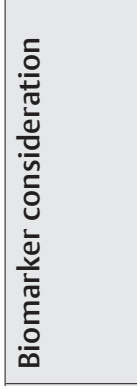 & $\frac{k}{z}$ & 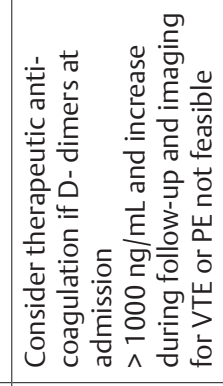 & $\frac{0}{2}$ & 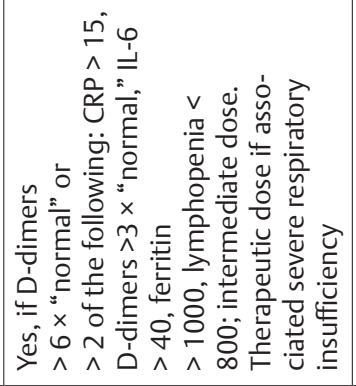 & $\frac{s}{z}$ & 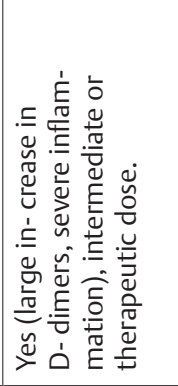 \\
\hline 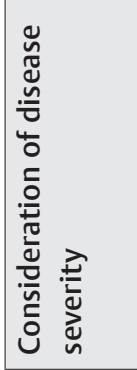 & $\frac{\mathbb{k}}{z}$ & $\frac{\mathbb{z}}{z}$ & $\frac{\Sigma}{z}$ & 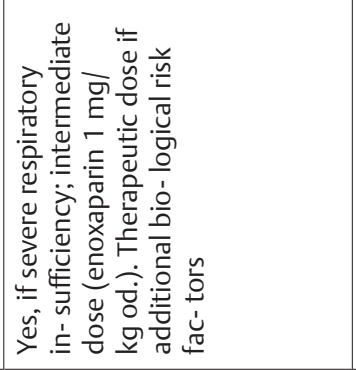 & $\frac{s}{z}$ & 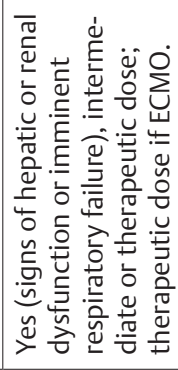 \\
\hline 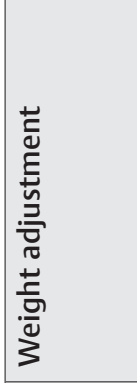 & $\frac{s}{z}$ & $\frac{s}{z}$ & 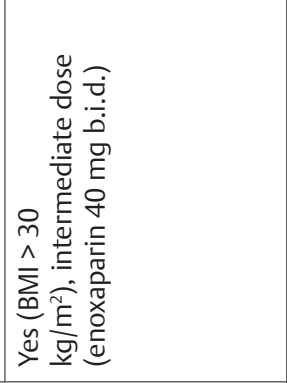 & 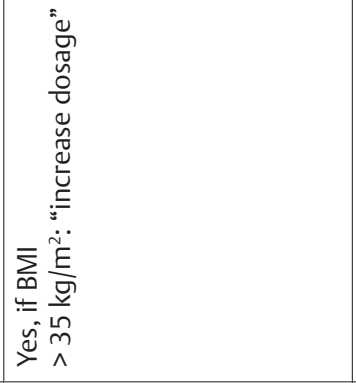 & $\frac{s}{z}$ & 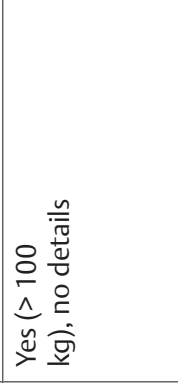 \\
\hline 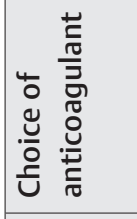 & 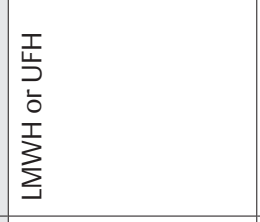 & $\frac{s}{z}$ & 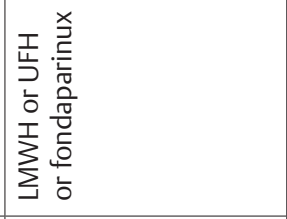 & 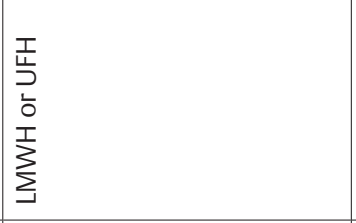 & 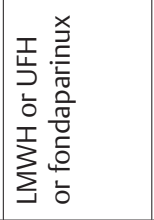 & 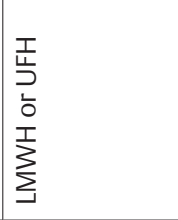 \\
\hline 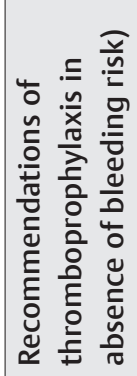 & 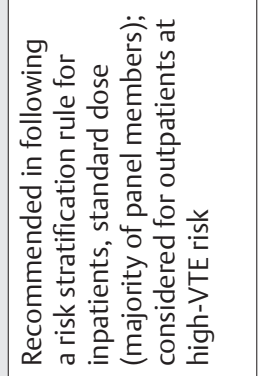 & 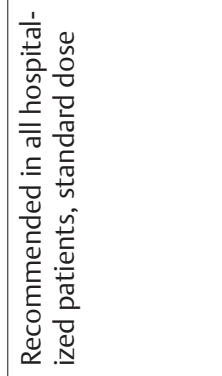 & 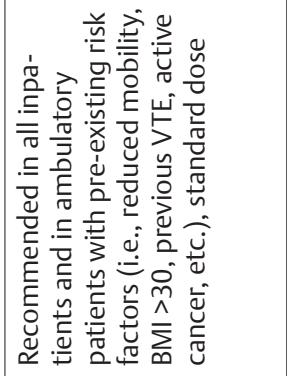 & 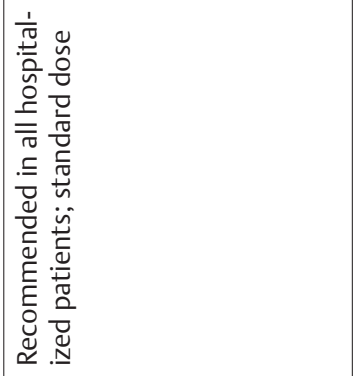 & 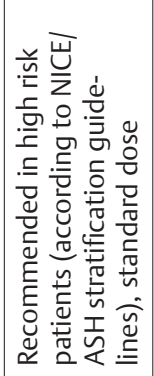 & 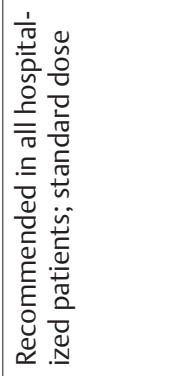 \\
\hline 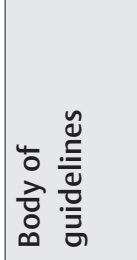 & 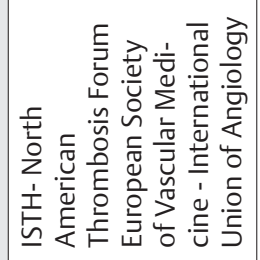 & 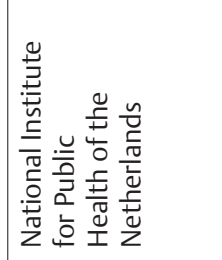 & 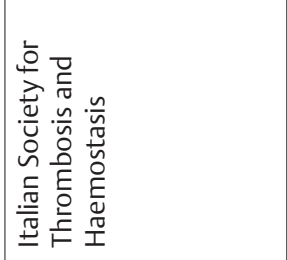 & 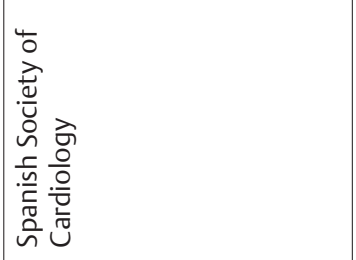 & 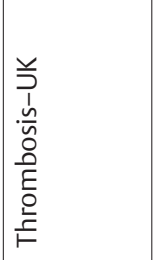 & 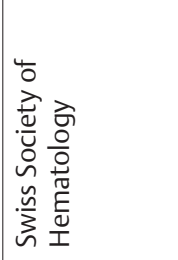 \\
\hline
\end{tabular}


assuming an increased predilection for developing VTE when they are COVID-19 positive. Hence, considering thromboprophylaxis in this subset of female patients, especially when they have other associated VTE risk factors, is advisable. - Table 2 shows the percentage of women with VTE events in the variant COVID-19 studies as of now. In a recent study from Italy ${ }^{37}$ published in mid July, persistent gender gap in occurrence VTE events was further emphasized. However, they believed an increased male predilection to be a representation of in-hospital population.Hence, occurrence of VTE in COVID-19 patients has a tendency to involve more men than women. Notwithstanding this gender gap, women deserve to be treated adequately for their VTE events with thromboprophylaxis as well as institute anticoagulant therapy when they get diseased by these events. Pregnancy and postpartum state in a COVID-19 positive patient deserve special attention, especially when they have other VTE risk factors.

\section{Conflicts of Interest}

None declared.

\section{References}

1 Borges do Nascimento IJ, Cacic N, Abdulazeem HM, et al. Novel Coronavirus infection (COVID-19) in humans: a scoping review and meta-analysis. J Clin Med 2020;9(4):941

2 La Vignera S, Cannarella R, Condorelli RA, Torre F, Aversa A, Calogero AE. Sex-specific SARS-CoV-2 mortality: among hormone-modulated ACE2 expression, risk of venous thromboembolism and hypovitaminosis D. Int J Mol Sci 2020;21(8):2948

3 Tang N, Li D, Wang X, Sun Z. Abnormal coagulation parameters are associated with poor prognosis in patients with novel coronavirus pneumonia. J Thromb Haemost 2020;18(4):844-847

4 Bikdeli B, Madhavan MV, Jimenez D, et al; Global COVID-19 Thrombosis Collaborative Group, endorsed by the ISTH, NATF, ESVM, and the IUA, Supported by the ESC Working Group on Pulmonary Circulation and Right Ventricular Function. COVID-19 and thrombotic or thromboembolic disease: implications for prevention, antithrombotic therapy, and follow-Up: JACC state-of-the-art review. J Am Coll Cardiol 2020;75(23):2950-2973

5 Lippi G, Plebani M. Laboratory abnormalities in patients with COVID-2019 infection. Clin Chem Lab Med 2020;58(7):1131-1134

6 Wang T, Chen R, Liu C, et al. Attention should be paid to venous thromboembolism prophylaxis in the management of COVID-19. Lancet Haematol 2020;7(5):e362-e363

7 World Health Organization. Clinical management of severe acute respiratory infection when novel coronavirus (2019-nCoV) infection is suspected. Interim guidance 28 January 2020

8 Bates SM, Rajasekhar A, Middeldorp S, et al. American Society of Hematology 2018 guidelines for management of venous thromboembolism: venous thromboembolism in the context of pregnancy. Blood Adv 2018;2(22):3317-3359

9 Royal College of Obstetricians and Gynaecologists. Reducing the risk of venous thromboembolism during pregnancy and the puerperium. Top Guideline No. 37a, 2015

10 Lippi G, Favaloro EJ. D-dimer is associated with severity of coronavirus disease 2019 (COVID- 19): a pooled analysis. Thromb Haemost 2020;120(5):876-878
11 Spyropoulos AC, Lipardi C, Xu J, et al. Improved benefit risk profile of rivaroxaban in a subpopulation of the MAGELLAN study. Clin Appl Thromb Hemost 2019;25:1076029619886022

12 Tang N, Bai H, Chen X, Gong J, Li D, Sun Z. Anticoagulant treatment is associated with decreased mortality in severe coronavirus disease 2019 patients with coagulopathy. J Thromb Haemost 2020;18(5):1094-1099

13 Ain DL, Albaghdadi M, Giri J, et al. Extra-corporeal membrane oxygenation and outcomes in massive pulmonary embolism: Two eras at an urban tertiary care hospital. Vasc Med 2018;23(1):60-64

14 Chinese Medical Doctor Association, Prevention and treatment of new coronavirus pneumonia associated venous thromboembolism, a Consensus statement (Preliminary Protocol). Chin Med J Netw2020:E007-E

15 Thachil J, Tang N, Gando S, et al. ISTH interim guidance on recognition and management of coagulopathy in COVID-19. J Thromb Haemost 2020;18(5):1023-1026

16 Practical guidance for the prevention of thrombosis and management of coagulopathy and disseminated intravascular coagulation of patients infected with COVID-19. Available at: https://thrombosisuk.org/downloads/ T\&H\%20and\%20COVID. pdf. Accessed April 29, 2020

17 Zhai Z, Li C, Chen Y, et al; Prevention Treatment of VTE Associated with COVID-19 Infection Consensus Statement Group. Prevention and Treatment of Venous Thromboembolism Associated with Coronavirus Disease 2019 Infection: A Consensus Statement before Guidelines. Thromb Haemost 2020;120(6):937-948

18 GFHT/GIHP Propositions pour le traitement anticoagulant pour la prévention du risque thrombotique chez un patient hospitalise avec COVID-19. Available at: https://site.geht.org/ docutheque/. Accessed April 29, 2020

19 Vivas D, Roldán V, Esteve-Pastor MA, et al. [Recommendations on antithrombotic treatment during the COVID-19 pandemic. Position statement of the Working Group on Cardiovascular Thrombosis of the Spanish Society of Cardiology]. Rev Esp Cardiol 2020;(e-pub ahead of print). doi: 10.1016/j. recesp.2020.04.006

20 Helms J, Tacquard C, Severac F, et al; CRICS TRIGGERSEP Group (Clinical Research in Intensive Care and Sepsis Trial Group for Global Evaluation and Research in Sepsis). High risk of thrombosis in patients with severe SARS-CoV-2 infection: a multicenter prospective cohort study. Intensive Care Med 2020;46(6):1089-1098

21 Oudkerk M, Büller HR, Kuijpers D, et al. Diagnosis, Prevention, and Treatment of Thromboembolic Complications in COVID-19: Report of the National Institute for Public Health of the Netherlands. Radiology 2020;(e-pub ahead of print). doi: https://doi.org/10.1148/radiol.2020201629

22 Casini A, Alberio L, Angelillo-Scherrer A, et al. Thromboprophylaxis and laboratory monitoring for in-hospital patients with COVID-19 - a Swiss consensus statement by the Working Party Hemostasis. Swiss Med Wkly 2020;150:w20247

23 COVID-19 and VTE/anticoagulation: frequently asked questions. Available at: https://www.hematology.org/covid-19/ covid-19-and-vte-anticoagulation. Accessed August 18, 2020

24 Recommendations for thrombosis prophylaxis in SARS-CoV-2 (COVID-19). Available at: http://gth-online.org/wp-content/ uploads/2020/04/GTH-Empfehlungen-COVID-19.pdf. Accessed April 29, 2020

25 Marietta M, Ageno W, Artoni A, et al. COVID-19 and haemostasis: a position paper from Italian Society on Thrombosis and Haemostasis (SISET) Blood Transfus 2020;18(3):167-169 
26 Bikdeli B, Madhavan MV, Jimenez D, et al; Global COVID-19 Thrombosis Collaborative Group, endorsed by the ISTH, NATF, ESVM, and the IUA, Supported by the ESC Working Group on Pulmonary Circulation and Right Ventricular Function. COVID-19 and thrombotic or thromboembolic disease: implications for prevention, antithrombotic therapy, and follow-up: JACC state-of-the-art review. J Am Coll Cardiol 2020;75(23):2950-2973

27 Ji H, Menini S, Zheng W, Pesce C, Wu X, Sandberg K. Role of angiotensin-converting enzyme 2 and angiotensin (1-7) in 17beta-oestradiol regulation of renal pathology in renal wrap hypertension in rats. Exp Physiol 2008;93(5):648-657

28 Dalpiaz PL, Lamas AZ, Caliman IF, et al. Sex hormones promote opposite effects on ACE and ACE2 activity, hypertrophy and cardiac contractility in spontaneously hypertensive rats. PLoS One 2015;10(5):e0127515

29 Walker RF, Zakai NA, MacLehose RF, et al. Association of testosterone therapy with risk of venous thromboembolism among men with and without hypogonadism. JAMA Intern Med 2019;180(2):190-197

30 Chang S, Christiansen $C F$, Bojesen A, Juul S, Münster AB, Gravholt $\mathrm{CH}$. Klinefelter syndrome and testosterone treatment: a national cohort study on thrombosis risk. Endocr Connect 2020;9(1):34-43

31 Zitzmann M, Nieschlag E. The CAG repeat polymorphism within the androgen receptor gene and maleness. Int J Androl 2003;26(2):76-83

32 Narayanan R, Coss CC, Dalton JT. Development of selective androgen receptor modulators (SARMs) Mol Cell Endocrinol 2018;465:134-142

33 Cui S, Chen S, Li X, Liu S, Wang F. Prevalence of venous thromboembolism in patients with severe novel coronavirus pneumonia. J Thromb Haemost 2020;18(6):1421-1424

34 Spiezia L, Boscolo A, Poletto F, et al. COVID-19-related severe hypercoagulability in patients admitted to intensive care unit for acute respiratory failure. Thromb Haemost 2020;120(6):998-1000

35 Ranucci M, Ballotta A, Di Dedda U, et al. The procoagulant pattern of patients with COVID-19 acute respiratory distress syndrome. J Thromb Haemost 2020;18(7):1747-1751
36 Fontana P, Casini A, Robert-Ebadi H, Glauser F, Righini M, Blondon M. Venous thromboembolism in COVID-19: systematic review of reported risks and current guidelines. Swiss Med Wkly 2020;150:w20301

37 Marone EM, Bonalumi G, Curci R, et al. Characteristics of venous thromboembolism in COVID-19 patients: a multicenter experience from Northern Italy. Ann Vasc Surg 2020. DOI:https://doi.org/10.1016/j.avsg.2020.07.007

38 Klok FA, Kruip M, van der Meer NJM, , et al. Incidence of thrombotic complications in critically ill ICU pa- tients with COVID-19. Thromb Res 2020;191:145-147

39 Klok FA, Kruip MJHA, van der Meer NJM, et al. Confirmation of the high cumulative incidence of thrombotic complications in critically ill ICU patients with COVID-19: An updated analysis. Thromb Res 2020;191:148-150

40 Llitjos JF, Leclerc M, Chochois C, et al. High incidence of venous thromboembolic events in anticoagulated severe COVID19 patients. J Thromb Haemost 2020. doi: http://dx.doi. org $/ 10.1111 /$ jth. 14869

41 Poissy J, Goutay J, Caplan M, et al. Lille ICU haemostasis COVID-19 group. Pulmonary embolism in COVID-19 patients: Awareness of an increased prevalence. Circulation 2020. doi: http://dx.doi.org/10.1161/IRCULA- TIONAHA.120.047430

42 Thomas W, Varley J, Johnston A, et al. Thrombotic complications of patients admitted to intensive care with COVID-19 at a teaching hospital in the United Kingdom. Thromb Res 2020;191:76-77

43 Grein J, Ohmagari N, Shin D, et al. Compassionate use of Remdesivir for patients with severe covid-19. N Engl J Med 2020;382(24):2327-2336

44 Grillet F, Behr J, Calame P, Aubry S, Delabrousse E. Acute pulmonary embolism associated with COVID-19 pneumonia detected by Pulmonary CT angiography. Radiology 2020:201544

45 Lodigiani C, Iapichino G, Carenzo L, et al. Humanitas COVID-19 task force. Venous and arterial thromboembolic complications in COVID-19 patients admitted to an academic hospital in Milan, Italy. Thromb Res 2020;191:9-14 